\title{
Binding of boar spermatozoa to oviductal epithelium in vitro in relation to sperm morphology and storage time
}

\author{
D Waberski, F Magnus, F Ardón, A M Petrunkina, K F Weitze and E Töpfer-Petersen \\ Institute for Reproductive Medicine, University of Veterinary Medicine Hannover, Foundation, Buenteweg 15, \\ D-30559 Hannover, Germany
}

Correspondence should be addressed to D Waberski; Email: Dagmar.Waberski@tiho-hannover.de

\begin{abstract}
In vitro short-term storage of boar semen for up to $72 \mathrm{~h}$ before insemination negatively affects fertility, but this often remains undetected during semen quality assessment. One important sperm function is the ability to form the functional sperm reservoir in the oviduct. In the present study, we used the modified oviductal explant assay to study sperm binding to oviductal epithelium in vitro in diluted boar semen stored for 24 or $72 \mathrm{~h}$. First, we determined the kinetics of in vitro sperm binding to oviductal epithelium in relation to co-incubation time of sperm and oviductal tissue pieces. Then, we studied how the binding of sperm to oviductal epithelium was affected by in vitro semen storage and by differences among individual boars. Sperm binding after different incubation times was significantly higher when semen was stored $24 \mathrm{~h}$ than after 72 -h storage $(P<0.05)$, and peaked at 30-90 min of incubation. Sperm binding differed between boars $(n=44)$, and was negatively correlated to the percentage of sperm with cytoplasmic droplets $(R=-0.51, P<0.001)$. There were no significant changes in motility, acrosome integrity and propidium iodide stainability during the 72 -h storage period. However, sperm-binding indices were significantly lower after $72 \mathrm{~h}$ in vitro storage than after $24-\mathrm{h}$ storage in sperm from boars with normal semen quality $(P<0.05)$; in contrast, the binding capacity of sperm from boars with higher percentages of morphologically altered sperm remained at a low level. The spermbinding capacity of sperm from four of the five boars with known subfertility was lower than the mean binding index minus one standard deviation of the boar population studied here. It is concluded that changes in the plasma membrane associated with in vitro ageing reduce the ability of stored boar sperm to bind to the oviductal epithelium. This study shows the potential of sperm-oviduct binding as a tool to assess both male fertility and changes in sperm function associated with in vitro ageing. Reproduction (2006) 131 311-318
\end{abstract}

\section{Introduction}

Selection of the functionally competent sperm population occurs during the sperm's route of fertilisation in the female tract. In most mammalian species, the uterotubal junction acts as a selective entrance filter for motile sperm with superior morphology. Upon reaching the oviduct, sperm are trapped in the intraluminal mucus of the caudal isthmus, where they bind to the ciliated epithelial cells that line the duct. The binding event creates in the female a sperm reservoir with a number of functions: control of sperm transport to the site of fertilisation, selection of viable sperm, maintenance of sperm viability and modulation of capacitation to synchronise sperm fertilising ability with the time of ovulation (reviewed recently by Scott 2000, Rodriguez-Martinez et al. 2001, Hunter 2002, Töpfer-Petersen et al. 2002). In mammalian species with a prolonged oestrous length, the ability of sperm to bind to the oviductal epithelium is an essential function that both ensures sperm survival and regulates capacitation until ovulation occurs. In the pig, sperm fertilising competence in the oviduct is maintained for up to $24 \mathrm{~h}$. However, it has been shown in vivo that the number of fertilising competent sperm in the oviduct is lowered by deficiencies in semen quality; these can be due either to individual characteristics of the boar or to ageing of the sperm during storage prior to insemination. More specifically, fewer viable sperm reach the oviductal isthmus, and viable sperm with decreased membrane stability have a shorter life span in the oviduct (Saacke et al. 1994, Waberski et al. 1994a). At present, there is no satisfactory explanation for the reduced fertility of stored boar semen. Within the first 3 days of semen preservation, the motility and acrosome integrity of sperm usually remain high, but various insemination trials indicate that the sperm suffer functional damage from storage conditions (Waberski et al. 1994a, Johnson et al. 2000). Sperm-oviduct binding assays have been used in various species, mostly to study the physiology of sperm-oviduct interaction and to determine the 
criteria for sperm selection. Numerous observations from in vivo and in vitro studies indicate that oviduct binding has a selective function that ensures that the sperm in the female tract are both morphologically and functionally vital (Hunter et al. 1987, Thomas et al. 1994, Mburu et al. 1997, Ellington et al. 1999, Gualtieri \& Talevi 2000, 2003). These observations have led to the hypothesis that decreased ability to form the functional sperm reservoir is due to deficiencies in sperm quality which either originate within the boar or are caused by in vitro storage, because such deficiencies reduce the capacity of the sperm to bind to the oviductal epithelium in vitro. In the present study, we used a modified oviductal explant assay (Petrunkina et al. 2001a) to characterise the sperm-oviduct binding capacity in diluted boar semen stored in vitro for 24 and $72 \mathrm{~h}$. We first established the kinetics of sperm binding to oviductal epithelium in relation to co-incubation time of sperm and oviductal tissue pieces (explants); then we characterised differences between boars and the effects of in vitro storage in diluted semen in terms of the capacity of sperm to bind to the endosalpingeal epithelium in vitro. The results indicate that, even after relatively short-term storage $(72 \mathrm{~h})$, it is semen ageing that leads to a reduced capacity of the sperm to bind to the oviductal epithelium in vitro. This effect is boar specific. Furthermore, the presence of sperm cytoplasmic droplets is concomitant with reduced binding ability. Moreover, the low binding index (BI) of sperm from boars known to be subfertile indicates that sperm-oviduct binding studies are potentially useful for the assessment of male fertility.

\section{Materials and Methods}

\section{Animals and semen}

Experiments were conducted at the Institute for Reproductive Medicine of the University of Veterinary Medicine Hannover, Foundation, Hannover, Germany.

In experiment 1, eight ejaculates of normal semen quality were used from four healthy, fertile, Large White and Pietrain boars from the institute's boar stud. The samples were diluted with the commercial boar semen extender Androhep (Waberski et al. 1994b) and tested after 24 and $72 \mathrm{~h}$ to evaluate the kinetics of oviductal explant-sperm binding.

For experiment 2, diluted semen was used from 44 healthy, fertile boars from three races - 28 Pietrain, 11 Hybrid (BHZP), three Large White, one German Landrace and one Duroc - from four German artificial insemination (AI) centres. Five boars were subfertile in relation to the average in the respective $\mathrm{Al}$ centre. Farrowing rate or litter sizes from these boars differed significantly from the mean farrowing rates and litter sizes obtained from a total of 150 boars in the respective $\mathrm{Al}$ centre. Data were calculated over a period of 9 months (October to July) with a customised fertility monitoring software programme (written by Dr H Brandt, University of Giessen). Exact fertility data were not available for the remaining 39 boars.
Semen tubes were packed in polystyrene boxes and delivered to the University of Veterinary Medicine Hannover within $24 \mathrm{~h}$ of collection. Three ejaculates each were used from 37 boars and two each from seven. Semen was evaluated macroscopically and microscopically according to standard sperm parameters, including motility of diluted semen, morphology of samples previously fixed in a formaldehyde citrate solution (Hancock 1956), and membrane integrity of samples stained by the supravital dye propidium iodide, as described by Harrison and Vickers (1990). Boars were divided into two groups according to sperm morphology. Group 1 comprised the boars with normal sperm morphology, defined as $<30 \%$ morphologically altered sperm and $<15 \%$ sperm with cytoplasmic droplets; group 2, those with $\geq 30 \%$ morphologically altered sperm or $\geq 15 \%$ with cytoplasmic droplets (Waberski et al. 1994b). Semen was stored at $17^{\circ} \mathrm{C}$ for $72 \mathrm{~h}$ in darkness. After 24 and $72 \mathrm{~h}$ of semen storage, sperm motility and acrosome integrity of sperm fixed in a formaldehyde citrate solution were evaluated, and semen samples were submitted to the oviductal explant assay.

\section{Sperm preparation}

Diluent was removed and motile sperm were selected through Percoll gradient density centrifugation (Vincent \& Nadeau 1984, Harrison et al. 1993). In a centrifuge tube, $4 \mathrm{ml}$ of $35 \%$ Percoll (Sigma-Aldrich, Deisenhofen, Germany) were layered on top of $2 \mathrm{ml}$ of $70 \%$ Percoll. Finally, $4 \mathrm{ml}$ diluted semen were added, with care taken to avoid mixing the solutions. The tubes were first centrifuged for $10 \mathrm{~min}$ at $300 \mathrm{~g}$, and then for $20 \mathrm{~min}$ at $700 \mathrm{~g}$. The sperm pellet was resuspended in $500 \mu \mathrm{l}$ modified Androhep without EDTA (Petrunkina et al. 2001a) and adjusted to $5 \times 10^{6} \mathrm{sperm} / \mathrm{ml}$. Motility and morphology were assessed before and after Percoll centrifugation.

\section{Oviductal explant assay}

Oviductal explants were prepared as previously described by Petrunkina et al. (2001a) from Fallopian tubes collected from sows from a local abattoir. In brief, the surrounding tissue was carefully removed and the tubes were opened longitudinally. Two pieces of $0.5-1 \mathrm{~mm}$ were cut from the longitudinal folds of the isthmic region of the oviductal epithelium and placed into modified Tyrode's balanced solution (TALP medium) (Parrish et al. 1988) containing $6 \mathrm{mg} / \mathrm{ml} \mathrm{BSA}$ and $2.2 \mathrm{mg} / \mathrm{ml}$ sodium pyruvate. The viability of the oviductal explants was assessed by evaluating their ciliary activity. Samples from two sows were used on each experimental day. After preparation of the two pairs of oviductal explants from each sow, one pair per sow was equilibrated in $60 \mathrm{ml}$ TALP medium at $39^{\circ} \mathrm{C}$ in a humidified atmosphere containing $5 \% \mathrm{CO}_{2}$. Sperm were added to the explants to a concentration of $1 \times 10^{5}$ cells and a final volume of $80 \mu \mathrm{l}$. In experiment 1, explants were divided into six groups, each consisting of a pair of 
explants as explained above. Sperm and explants were co-incubated for 5, 15, 30, 60, 90 or $120 \mathrm{~min}$ in TALP medium at $39^{\circ} \mathrm{C}$ and in $5 \% \mathrm{CO}_{2}$. In experiment 2, several groups of sperm and explants were successively co-incubated for $15 \mathrm{~min}$ each. The co-incubation time of $15 \mathrm{~min}$ was used because the results of experiment 1 showed that boar differences were greatest at this point in time, when none of the samples had yet passed its maximal sperm BI. Explants were freed of loosely attached sperm by vigorously washing twice in TALP medium. They were then transferred to prewarmed slides, covered with cover-slips supported by silicon grease and analysed by videomicrography, as previously described (Petrunkina et al. 2001a). Three separate fields from both explants of a pair were videotaped (Panasonic video monitor WV-BM 1400; Kappa video camera CF8/1); the image was constantly refocused so that all sperm cells could be clearly seen and counted. Bound sperm were counted at the edges and surfaces of the explants by direct observation. As the geometric shape of the videotaped fields with bound sperm differed between regions, the surface of the fields also varied. To take into account the variation in surface area and the heterogeneity of sperm binding, the surface area was included in the quantification of binding. The surface area of each particular explant region observed here was calculated from the videotape by image analysis software (Mika Medical, Version 2.0, Rosenheim, Germany). The number of bound sperm per $0.01 \mathrm{~mm}^{2}$ surface area was defined for each explant within the pair as the sum of the number of sperm bound to each region divided by the sum of the areas of the three fields. The $\mathrm{BI}$ for each explant was determined by the following formula: $B I_{E}=\left(N_{R 1}+N_{R 2}+N_{R 3}\right) /\left(S_{R 1}+S_{R 2}+\right.$ $\left.S_{R 3}\right)$. Then, the arithmetic mean was calculated for each pair of explants. The $\mathrm{BI}$ for each sperm sample was obtained by calculating the arithmetic mean of the $\mathrm{BI}$ of the two explant pairs.

\section{Statistical analyses}

The $\mathrm{BI}$ and the conventional spermatological parameters were analysed with a statistical program package (SAS Institute, Cary, NC, USA). Data are presented as mean \pm S.D. In experiment 1 , the effects of the co-incubation time and of the storage time on the sperm binding capacity were evaluated by the Wilcoxon test. In experiment 2, the influence of the storage time and the sperm morphology groups on the $\mathrm{BI}$ was estimated by two-factorial analysis of variance (procedure GLM). Pearson correlation analysis (procedure CORR) was used to determine the relationship between $\mathrm{Bl}$ and sperm parameters. The criterion for statistical significance was $P<0.05$.

\section{Results}

\section{Experiment 1}

\section{Kinetics of oviductal explant-sperm binding}

The number of sperm bound to the oviduct $(\mathrm{BI})$ varied with time, reaching a peak at 30-90 min for semen stored for $24 \mathrm{~h}$ and at $30 \mathrm{~min}$ for semen stored for $72 \mathrm{~h}$. After 120-min incubation, the $\mathrm{BI}$ decreased significantly in both semen groups. The $\mathrm{BI}$ at 5, 15 and 90 min of incubation of semen stored for $24 \mathrm{~h}$ was significantly higher $(P<0.05)$ than that of semen stored for $72 \mathrm{~h}$ (Fig. 1).

\section{Experiment 2}

\section{Semen parameters}

A total of 118 ejaculates were evaluated from 44 boars. Two ejaculates showed a motility of $<70 \%$ and were therefore excluded from experiments. Sperm morphology was normal for 30 boars, which were classified as group 1 . The 14 boars with high percentages of cytoplasmic droplets $(n=9)$ and/or high percentages of total morphologically altered sperm were classified as group 2. Motility and acrosome integrity did not differ significantly at $24-72 \mathrm{~h}$ of storage.

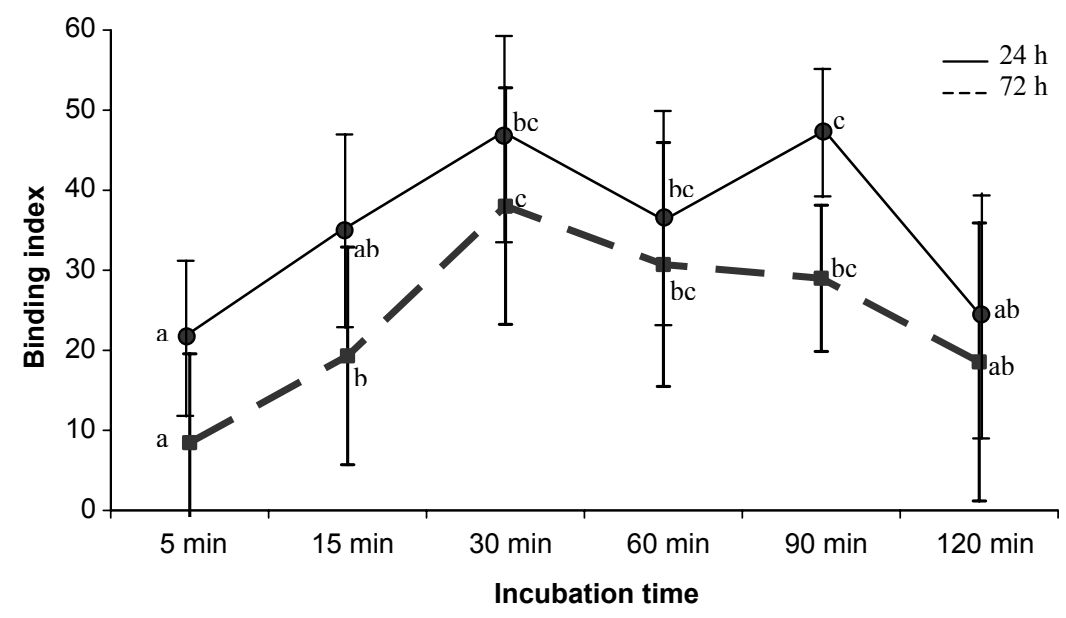

www.reproduction-online.org
Figure 1 Kinetics of sperm binding to oviductal explants in semen stored for 24 and $72 \mathrm{~h}$. Binding index (BI): number of sperm bound per $0.01 \mathrm{~mm}^{2}$ (means \pm S.D.). a-C: Significant differences between incubation times $(P<0.05) . n=2$ explants each for eight ejaculates from four boars per incubation time and in vitro semen storage period ( 24 and $72 \mathrm{~h}$ ). 
Percoll centrifugation significantly influenced sperm motility and the percentage of morphologically altered sperm but did not influence the percentage of sperm with cytoplasmic droplets (Table 1).

\section{Relation between sperm morphology, storage time and $\mathrm{BI}$}

The average explant size was $7.3 \pm 1.2 \mathrm{~mm}^{2}$; there was no influence of sow or explant size on the BI. Binding indices were based on a fixed co-incubation time for sperm and explants of $15 \mathrm{~min}$. After 24-h sperm storage, the mean $\mathrm{BI}$ of all boars was $26.4 \pm 8.9$, ranging from 21.1 to 44.0 . The mean $\mathrm{BI}$ differed between boars and is presented with indication of the morphology groups in Fig. 2. The $\mathrm{Bl}$ was significantly $(P<0.001)$ negatively correlated to the percentage of total morphologically altered sperm and to the percentage of cytoplasmic droplets (Fig. 3). There was no significant correlation between $\mathrm{BI}$ and motility or percentage of viable sperm after staining with propidium iodide after either 24 or $72 \mathrm{~h}$ of storage.

After $72 \mathrm{~h}$ of semen storage time in vitro, the mean $\mathrm{BI}$ of all boars was significantly $(P<0.001)$ lower than that of semen stored for $24 \mathrm{~h}$ (20.1 vs 26.4). In group 1, boars with normal sperm morphology, semen storage had a significant $(P<0.001)$ negative effect on the $\mathrm{BI}$. Semen storage did not show an effect $(P>0.05)$ on the $\mathrm{BI}$ of group 2, the boars with impaired sperm morphology. After 24 and $72 \mathrm{~h}$ of semen storage, the $\mathrm{BI}$ of group 1 was significantly higher $(P<0.001)$ than that of group 2 (Fig. 4).

\section{Standard sperm parameters and sperm binding in subfertile boars}

Sperm motility was normal in all subfertile boars. In four of these boars (nos. 37-40), which belonged to group 2, percentages of morphologically abnormal sperm were elevated. The fifth boar (no. 36) was classified as group 1, but its sperm morphology was close to the threshold for group 2. The sperm of this specific boar had 16\% tail loops before Percoll centrifugation. The BI of the sperm of

Table 1 Sperm quality before and after Percoll centrifugation $(n=28$ ejaculates from 9 boars).

\begin{tabular}{lcc}
\hline Sperm parameter & Control & $\begin{array}{c}\text { After Percoll } \\
\text { centrifugation }\end{array}$ \\
\hline Motility (\%) & $76.4^{\mathrm{a}}$ & $82.3^{\mathrm{b}}$ \\
Morphologically altered sperm (\%) & $30.8^{\mathrm{a}}$ & $25.1^{\mathrm{a}}$ \\
Cytoplasmic droplets (\%) & $13.1^{\mathrm{a}}$ & $12.8^{\mathrm{a}}$ \\
Other alterations (\%) & $8.0^{\mathrm{a}}$ & $4.4^{\mathrm{b}}$ \\
Propidium iodide positive (\%) & $17.2^{\mathrm{a}}$ & $16.2^{\mathrm{a}}$ \\
\hline
\end{tabular}

a,b. different superscripts in a single row indicate significant differences $(P<0.05)$ within that row. all four of these group 2 boars was lower than the mean $\mathrm{BI}$ minus one standard deviation of the population. The BI of boar no. 36 was similar to the mean $\mathrm{BI}$ of all boars (Table 2).

\section{Discussion}

The present study shows that both in vitro storage of diluted boar semen and the prevalence of cytoplasmic droplets may reduce the binding capacity of sperm to the oviductal epithelium in vitro. A modified sperm-oviduct binding assay (Petrunkina et al. 2001a), originally based on the oviductal explant assay of Suarez et al. (1991), was used to quantify sperm binding to oviductal epithelium in vitro. The assay is characterised by the use of fresh tissue pieces recovered from the isthmic endosalpingeal epithelium of sows immediately after slaughter and shortterm incubation of defined sperm numbers with these oviductal explants. The absence of culture techniques diminishes dedifferentiation of the epithelium morphology, thereby rendering the assay closer to the in vivo situation than tissue culture systems. In the present study, Percoll centrifugation was used for efficient removal of extender, seminal plasma, free cytoplasmic droplets and debris (Petrunkina et al. 2004a) instead of swim-up, as described previously (Petrunkina et al. 2001a). Concomitantly, sperm were to a certain extent selected for superior motility, but there was no significant selection for morphology. The latter applied mostly to tail loops, but not to cytoplasmic droplets and plasma membrane integrity. There is evidence from a number of in vivo studies that no major amount of seminal plasma or extender may pass through the narrow lumen of the uterotubal junction of pigs in oestrus (Mann et al. 1956, Hunter et al. 1972). Immotile sperm with severely abnormal sperm morphology, such as tail loops, are also hindered in their entrance in the oviduct in vivo (Hunter 1988). Therefore, the Percoll washing of sperm contributed to the establishment of standardised assay conditions similar to the in vivo situation. There are two reasons for the replacement of swim-up by Percoll washing: first, more sperm are harvested through Percoll centrifugation than with swim-up. Second, sperm are usually incubated for the swim-up procedure for $1 \mathrm{~h}$ at $39^{\circ} \mathrm{C}$ in a medium containing bicarbonate, which may induce capacitation-like destabilisation processes in sperm membranes, especially in long-term stored semen, whereas Percoll washing is unlikely to accelerate capacitation (Suzuki et al. 1995).

The time variation study showed that sperm-oviduct binding depended on the co-incubation time of sperm with oviductal explants; this occurred in a boar-specific and semen-age-dependent manner. Sperm binding reached its maximum after $30 \mathrm{~min}$ of incubation regardless of semen age, and may have been limited by the capacity of sperm membranes to interact with the oviductal epithelium in vitro. The decreased number of bound 


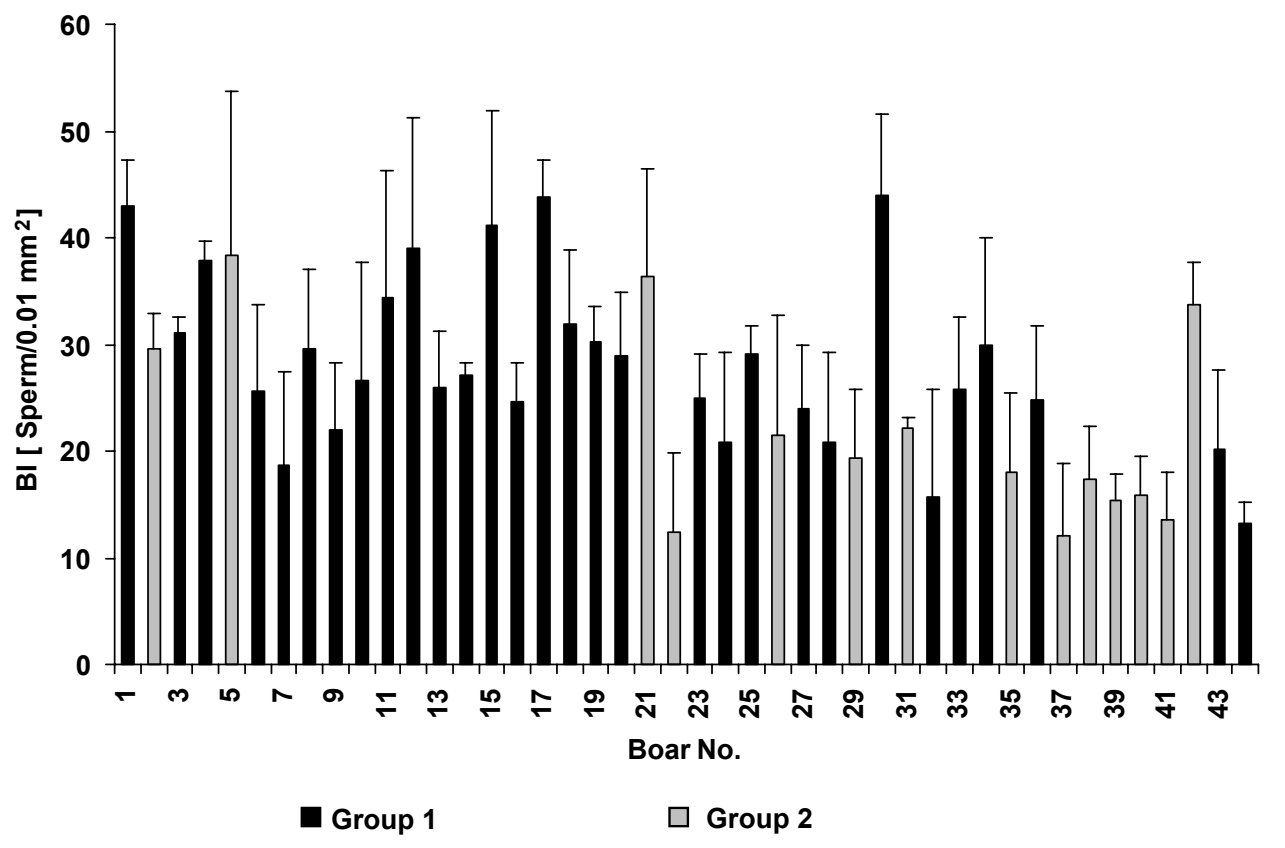

Figure 2 Sperm binding index (BI at 15-min incubation time; means \pm S.D.) in the oviductal explant assay after 24-h in vitro semen storage of semen from boars with normal sperm morphology (group 1, $n=30$ ) and from those with high percentages of morphologically altered sperm (group 2, $n=14)$.

sperm after 120-min incubation was probably the result of two independent phenomena: the capacity of sperm to bind to the oviductal epithelium, and their ability to respond to the capacitating effect of the incubation medium, the latter resulting in continuing release of bound sperm. Although binding of sperm to oviductal cells in vitro suppresses tyrosine phosphorylation of sperm membrane proteins and cytosolic $\mathrm{Ca}^{2+}$ intake (Dobrinski et al. 1996, Fazeli et al. 1999, Petrunkina et al. 2001b, 2003), bound sperm may gradually capacitate and eventually be released from oviduct epithelial cells. Similar sperm binding profiles have recently been shown for fresh dog spermatozoa in a homologous oviductal explant assay (Petrunkina et al. 2004b). In that assay, maximal binding density was reached after 90-min co-incubation of the sperm with oviductal explants. The difference in the incubation length associated with the highest $\mathrm{BI}$ in the two species indicates that the resistance to in vitro capacitation is higher in fresh dog sperm than in stored boar sperm. Capacitation has been shown in various species to reduce homologous in vitro sperm-oviduct binding (Thomas et al. 1995, Lefebvre \& Suarez 1996, Fazeli et al. 1999). The reduced binding capacity of boar sperm stored for $72 \mathrm{~h}$ observed in this study may be the reason why fertility rates were lower than those of fresh semen. The reduced binding observed here is probably due to changes in boar sperm membrane that are associated with storage. Dilution and cooling induce phase events and the concomitant entrance of free calcium ions from the environment into the sperm; these pheonomena could stimulate the calcium-dependent processes associated with capacitation (Watson 1996). There is evidence from in vitro studies that the binding of sperm to the oviductal epithelium is preferential for viable sperm with low $\mathrm{Ca}^{2+}$ concen- trations and suppressed protein phosphorylation (Dobrinski et al. 1996, Petrunkina et al. 2001b). A similar preferential binding mechanism for membrane-stable sperm in fresh boar semen may be responsible for the differences in the binding capacity of stored boar sperm observed here. In boars with higher percentages of morphologically altered sperm, binding was constantly lower during the 72 -h storage period. The decrease in binding capacity due to storage influences was most evident in semen from boars with good sperm morphology. Neither motility nor acrosome integrity was affected, indicating that these standard semen parameters are insufficient for the detection of the effect of short-term storage on liquid boar semen.

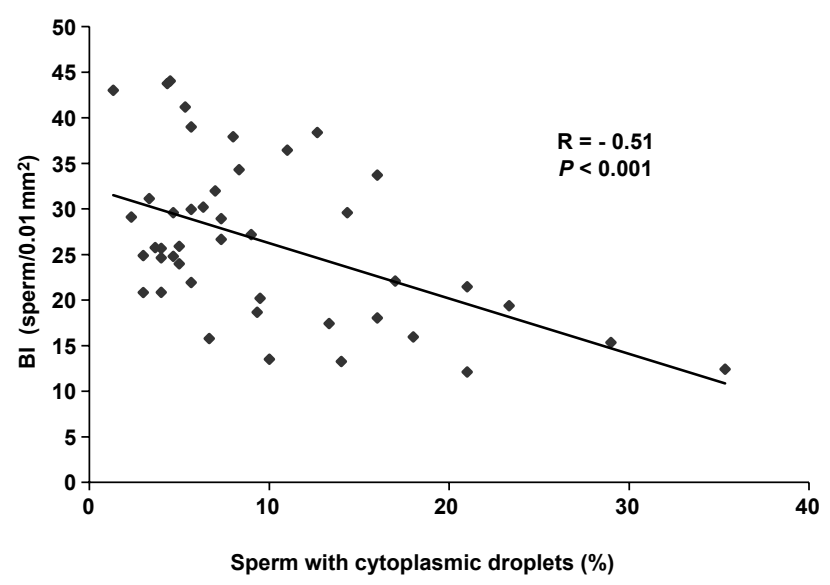

Figure 3 Correlation between the binding index ( $\mathrm{BI}$ at 15-min incubation time) in the oviductal explant assay and the percentage of sperm with cytoplasmic droplets after 24-h in vitro semen storage ( $n=44$ boars). 


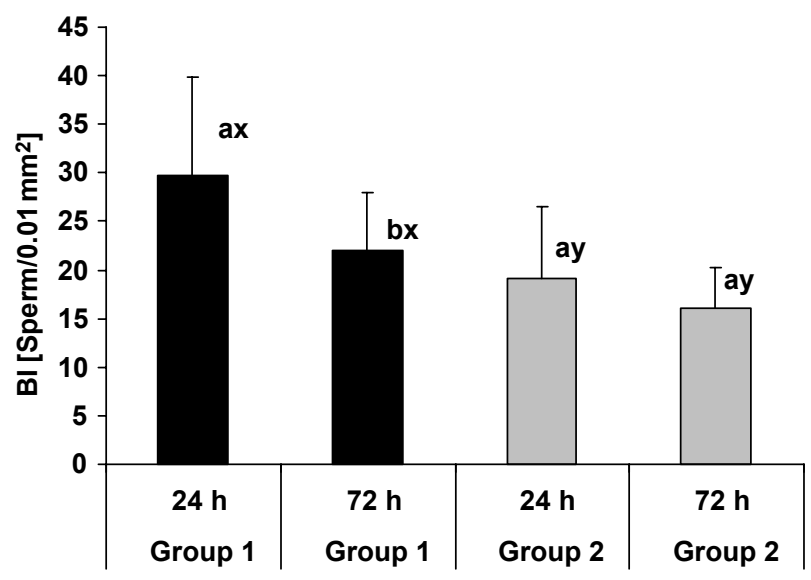

Figure 4 Sperm binding index ( $\mathrm{BI}$ at 15-min incubation time) in the oviductal explant assay after 24- and 72-h in vitro semen storage in boars with normal sperm morphology (group 1, $n=30$ ) and with high percentages of morphologically altered sperm (group 2, $n=14$ ). $a$, b: significant differences between semen storage times within boar groups $(P<0.001) ; \mathrm{x}, \mathrm{y}$ : significant differences between boar groups after same semen storage time $(P<0.001)$.

Even with advanced spermatological testing for boar sperm responsiveness to in vitro capacitation conditions (with parameters such as changes in membrane integrity, response to $\mathrm{Ca}^{2+}$ ionophore, and internal $\mathrm{Ca}^{2+}$ content), it was not possible to detect the changes in sperm quality associated with semen storage for up to $72 \mathrm{~h}$ (Conejo-Nava et al. 2003, Petrunkina et al. 2005a). Furthermore, it is known that the functional ability to regulate sperm volume under iso- and hypotonic conditions is not affected by 48-h in vitro storage (Petrunkina et al. 2005b). On the other hand, the results of the present study indicate that the oviductal explant assay is a sensitive functional test for the detection of the effects of in vitro ageing in boar sperm stored for a short time. However, the molecular background of this observation remains unclear. Sperm-oviduct binding is mediated by lectin-like secretory proteins from the male genital tract, which become associated to the sperm surface during ejaculation (Töpfer-Petersen 1999, Suarez 2002). In pigs, complex mannose structures are involved in the carbohydrate-based initial sperm-oviduct binding mechanism (Wagner et al. 2002). Changes in the plasma membrane and/or associated surface proteins during in vitro ageing may alter the function of the receptor-ligand system, thus reducing the ability of sperm to communicate with the oviductal epithelium. In spite of the use of so-called long-term extenders, boar sperm gradually begin losing their fertilisation capacity from the first day of storage. Thus, the prevention of in vitro ageing through modified preservation techniques remains a challenge that requires sensitive assay systems to monitor storage-associated changes.

Like in vitro ageing, the presence of increased amounts of cytoplasmic droplets was also associated with reduced sperm binding capacity. Although there was some binding of sperm with cytoplasmic droplets to explants, boars whose ejaculates had a high percentage of such morphological alteration had a lower $\mathrm{Bl}$, which is evidence that a certain stage of maturation or morphological integrity is needed for sperm to bind to the oviductal epithelium (Petrunkina et al. 2001a). However, it is not clear whether the reduced binding capacity in ejaculates containing increased numbers of cytoplasmic droplets applies only to this particular sperm subpopulation, or whether it represents a general feature equally distributed over the entire sperm population. Clarification of this question could help to determine whether increasing sperm numbers could compensate for this specific morphological defect. At present, it is not known whether and to what extent sperm with cytoplasmic droplets may enter the oviduct through the barriers of the uterotubal junction. The absence of these droplets in electron microscopic pictures would not be strong evidence, as cytoplasmic droplets could have been shed during their passage through the mechanical barriers in the female tract ( $\mathrm{R} \mathrm{H} \mathrm{F} \mathrm{Hunter,} \mathrm{personal} \mathrm{com-}$ munication). In vivo trials indicate that the presence of more than $15 \%$ cytoplasmic droplets is associated with reduced fertility (Waberski et al. 1994b). While cytoplasmic droplets are the most frequently observed defects in boar ejaculates, the next most frequent ones, tail loops and other tail shape abnormalities, are deselected in vivo by the barriers of the uterotubal junction. Those sperm will therefore not come into contact with the oviductal epithelium in vivo, so that binding studies for these specific defects are senseless. However, such sperm are

Table 2 Sperm parameters and binding index in the oviduct explant assay in five subfertile boars (nos. 36-40) in relation to the means of the boar population ( $n=44$ boars). Data shown per boar are means of three ejaculates stored for $24 \mathrm{~h}$.

\begin{tabular}{|c|c|c|c|c|c|c|}
\hline \multirow[b]{2}{*}{ Sperm parameter } & \multicolumn{5}{|c|}{ Boar no. } & \multirow{2}{*}{$\begin{array}{c}\text { Mean } \pm \text { S.D. } \\
(n=44 \text { boars })\end{array}$} \\
\hline & 36 & 37 & 38 & 39 & 40 & \\
\hline Morphologically altered sperm (\%) & 29.0 & $42.0 *$ & $44.2 *$ & $40.8^{*}$ & 32.2 & $24.3 \pm 10.6$ \\
\hline Sperm with cytoplasmic droplets (\%) & 4.2 & $20.5 *$ & 14.3 & $28.0 *$ & $18.2 *$ & $9.8 \pm 7.5$ \\
\hline Propidium iodide-positive sperm (\%) & $10.0 *$ & $10.3 *$ & 11.8 & $7.7 *$ & $9.3 *$ & $14.5 \pm 5.1$ \\
\hline Binding index ${ }^{1}$ & 24.8 & $12.1 *$ & $17.4 *$ & $15.3 *$ & $16.0 *$ & $26.4 \pm 8.9$ \\
\hline
\end{tabular}

* Value lies beyond the range (mean \pm S.D.) of the boar population.

${ }^{1}$ Sperm binding index in the oviduct explant assay at 15 -min incubation time. 
selected in vitro by Percoll centrifugation. Boar 36 had high percentages of sperm with tail loops and was subfertile, but its BI was normal, due to the fact that this defect was eliminated by Percoll centrifugation before the sample was submitted to the oviductal explant assay. Clearly, the presence of tail loops does not negatively affect sperm binding capacity in the rest of the sperm population; therefore, increasing sperm numbers in the insemination dose can in fact compensate for the possible effect of tail loops on the formation of the oviductal sperm reservoir.

It is likely that the binding differences observed here indicate differences in fertilisation capacity. Whether this is ultimately reflected in reduced farrowing rate and litter sizes depends largely on the insemination dose, intervals between $\mathrm{Al}$ and ovulation and other farm management factors (Waberski et al. 1994a, Johnson et al. 2000). The present study was not designed to find a correlation between $\mathrm{BI}$ and fertility, and it cannot be expected that there would be a linear relationship for any sperm parameter to be tested. However, there was a relationship between impaired sperm-oviduct binding capacity in vitro and subfertility in a few boars of this study. The prediction of subfertility is a primary goal in sperm assessment of domestic animal species. There is support for the oviduct binding assay as a source of valuable information about fertility-relevant sperm function: a study on bulls showed a positive association between sperm-oviduct binding in vitro and non-return rates (De Pauw et al. 2002). The study of sperm-oviduct binding with the oviduct explant assay fulfils the criteria for an ideal assay system which tests for multiple traits on sperm; that is, sperm binding of viable, morphologically intact spermatozoa (Hammerstedt 1996). This assay evaluates an important function of the plasma membrane, which is the key organelle to be tested in assays of sperm quality (Harrison 1997). In spite of the limitations of an in vitro system for the study of spermoviduct interaction, due to the lack of the dynamic local microenvironment in the Fallopian tube (Hunter \& Rodriguez-Martinez 2002, Hunter 2003), it seems that relevant parts of the sperm-oviduct binding capacity can be measured by the oviductal explant assay. Impaired binding ability may reflect sperm deficiencies that prevent the establishment of the functional sperm reservoir in the female reproductive tract. The continuing identification of relevant receptor-ligand systems of sperm-oviduct binding may eventually lead to new perspectives for a practical assay designed to test for defined sperm characteristics relevant to fertility.

\section{Acknowledgements}

This study was supported by the Association of German Pig Production (ZDS e.V., Bonn, Germany), Minitüb GmbH (Landshut, Germany) and BioPore Inc (State College, PA, USA). We also thank GFS Ascheberg and Dr Horst Brandt (University of Giessen, Germany) for providing fertility data and for the generous donation of boar semen, and Rodney Chinn and Judith McAlister-Hermann, PhD, for critical review of the English text. The authors declare that there is no conflict of interest that would prejudice the impartiality of this scientific work.

\section{References}

Conejo-Nava J, Fierro R, Gutierrez CG \& Betancourt M 2003 Membrane status and in vitro capacitation of porcine sperm preserved in long-term extender. Archives of Andrology 49 287-295.

De Pauw IMC, Van Soom A, Laevens H, Verberckmoes S \& De Kruif A 2002 Sperm binding to epithelial explants in bulls with different non-return rates investigated with a new in vitro model. Biology of Reproduction 67 1073-1079.

Dobrinski I, Suarez SS \& Ball BA 1996 Intracellular calcium concentration in equine spermatozoa attached to oviductal epithelial cells in vitro. Biology of Reproduction 54 783-788.

Ellington JE, Evenson DP, Wright RW Jr, Jones AE, Schneider CS, Hiss GA \& Brisbois RS 1999 Higher quality human sperm in a sample selectively attached to oviduct (Fallopian tube) epithelial cells in vitro. Fertility and Sterility 71 924-929.

Fazeli A, Duncan AE, Watson PF \& Holt WV 1999 Sperm-oviduct interaction: induction of capacitation and preferential binding of uncapacitated spermatozoa to oviductal epithelial cells in porcine species. Biology of Reproduction 60 879-886.

Gualtieri R \& Talevi R 2000 In vitro-cultured bovine oviductal cells bind acrosome-intact sperm and retain this ability upon sperm release. Biology of Reproduction 62 1754-1762.

Gualtieri R \& Talevi R 2003 Selection of highly fertilizationcompetent bovine spermatozoa through adhesion to the Fallopian tube epithelium in vitro. Reproduction 125 251-258.

Hammerstedt RH 1996 Evaluation of sperm quality: identification of the subfertile male and courses of action. Animal Reproduction Science 42 77-87.

Hancock JL 1956 The morphology of boar spermatozoa. Journal of the Royal Microscopical Society 76 84-97.

Harrison RAP 1997 Sperm plasma membrane characteristics and boar semen fertility. Journal of Reproduction and Fertility Supplement 52 195-211.

Harrison RAP \& Vickers SE 1990 Use of fluorescent probes to assess membrane integrity in mammalian spermatozoa. Journal of Reproduction and Fertility 88 343-352.

Harrison RAP, Mairet B \& Miller NGA 1993 Flow cytometric studies of bicarbonate $\mathrm{Ca}^{2+}$ influx in boar sperm populations. Molecular Reproduction and Development 35 197-208.

Hunter RHF, Fléchon B \& Fléchon JE 1987 Pre- and peri-ovulatory distribution of viable spermatozoa in the pig oviduct: a scanning electron microscope study. Tissue and Cell 19 423-436.

Hunter RHF 1988 In The Fallopian Tubes: Their Role in Fertility and Infertility, pp 53-80. Berlin: Springer-Verlag.

Hunter RHF 2002 Vital aspects of Fallopian tube physiology in pig. Reproduction in Domestic Animals 37 186-190.

Hunter RHF 2003 Reflections upon sperm-endosalpingeal and sperm-zona pellucida interactions in vivo and in vitro. Reproduction in Domestic Animals 38 147-154.

Hunter RHF \& Rodriguez-Martinez H 2002 Analysing mammalian fertilisation: reservations and potential pitfalls with an in vitro approach. Zygote 10 11-15.

Hunter RHF, Karagiannidis A \& Mann T 1972 Fate of seminal plasma after intrauterine insemination in the pig. Journal of Reproduction and Fertility 29 303-305.

Johnson LA, Weitze KF, Fiser P \& Maxwell WMC 2000 Storage of boar semen. Animal Reproduction Science 62 143-172.

Lefebvre R \& Suarez SS 1996 Effect of capacitation on bull sperm binding to homologous oviductal epithelium. Biology of Reproduction 54 575-582. 
Mann T, Polge C \& Rowson LEA 1956 Participation of seminal plasma during the passage of spermatozoa in the female tract of the pig and horse. Journal of Endocrinology 13 133-140.

Mburu JN, Rodriguez-Martinez H \& Einarsson S 1997 Changes in sperm ultrastructure and localisation in the porcine oviduct around ovulation. Animal Reproduction Science 47 137-148.

Parrish JJ, Susko-Parrish J, Winer MA \& First NL 1988 Capacitation of bovine sperm by heparin. Biology of Reproduction 38 1171-1180.

Petrunkina AM, Gehlhaar R, Drommer W, Waberski D \& TöpferPetersen E 2001a Selective sperm binding to pig oviductal epithelium in vitro. Reproduction 121 889-896.

Petrunkina AM, Friedrich J, Nolte A, Drommer W, Bicker G, Waberski D \& Töpfer-Petersen E 2001b Kinetic characterization of the changes in tyrosin phosphorylation of membrane proteins, cytosolic $\mathrm{Ca}^{2+}$ concentrations and viability in boar sperm populations selected by binding to oviductal epithelial cells. Reproduction 122 469-480.

Petrunkina AM, Simon K, Günzel-Apel AR \& Töpfer-Petersen E 2003 Regulation of capacitation of canine spermatozoa during co-culture with heterologous oviductal epithelial cells. Reproduction in Domestic Animals 38 455-463.

Petrunkina AM, Radcke S, Günzel-Apel AR, Harrison RAP \& TöpferPetersen E 2004a Role of potassium channels, the sodium-potassium pump and the cytoskeleton in the control of dog sperm volume. Theriogenology 61 35-54.

Petrunkina AM, Simon K, Günzel-Apel AR \& Töpfer-Petersen E $2004 b$ Kinetics of protein tyrosine phosphorylation in sperm selected by binding to homologous and heterologous oviductal explants: how specific is the regulation by the oviduct? Theriogenology 61 1617-1634.

Petrunkina AM, Volker G, Weitze KF, Beyerbach M, Töpfer-Petersen E \& Waberski D 2005a Detection of cooling-induced membrane changes in the response of boar sperm to capacitating conditions. Theriogenology 63 2278-2299.

Petrunkina AM, Jebe E \& Töpfer-Petersen E 2005b Regulatory and necrotic volume increase in boar spermatozoa. Journal of Cellular Physiology 204 508-521.

Rodriguez-Martinez H, Tienthai P, Suzuki K, Funahashi H, Ekwall H \& Johannisson A 2001 Involvement of oviduct in sperm capacitation and oocyte development in pigs. Reproduction Supplement 58 129-145.

Saacke RG, Nadir S \& Nebel RL 1994 Relationship of semen quality to sperm transport, fertilization and embryo quality in ruminants. Theriogenology $\mathbf{4 1}$ 45-49.

Scott MA 2000 A glimpse at sperm function in vivo: sperm transport and epithelial interaction in the female reproductive tract. Animal Reproduction Science 60-61 337-348.
Suarez SS 2002 Carbohydrate-mediated formation of the oviductal sperm reservoir in mammals. Cells, Tissues, Organs 168 105-112.

Suarez SS, Redfern K, Raynor P, Martin F \& Phillips DM 1991 Attachment of boar sperm to mucosal explants of oviduct in vitro: possible role in formation of a sperm reservoir. Biology of Reproduction 44 998-1004.

Suzuki K, Ebihara M, Nagai T \& Harrison RAP 1995 Ejaculated boar spermatozoa can penetrate oocytes maturing in vitro without preincubation. Journal of Reproduction and Development 41 229-233.

Thomas PGA, Ball BA \& Brinsko SP 1994 Interaction of equine spermatozoa with oviduct cell explants is affected by estrous cycle and anatomic origin of explant. Biology of Reproduction 51 222-227.

Thomas PGA, Ball BA \& Brinsko SP 1995 Changes associated with induced capacitation influence the interaction between equine spermatozoa and oviduct epithelial cell monolayers. Biology of Reproduction Monographs 1 697-705.

Töpfer-Petersen E 1999 Carbohydrate-based interactions on the route of a spermatozoon to fertilization. Human Reproduction 5 314-329.

Töpfer-Petersen E, Wagner A, Friedrich J, Petrunkina A, Ekhlasi-Hundrieser M, Waberski D \& Drommer W 2002 Function of the mammalian oviductal sperm reservoir. Journal of Experimental Zoology 292 210-215.

Vincent R \& Nadeau D 1984 Adjustment of osmolality of Percoll for the isopycnic separation of cells and cell organelles. Analytic Biochemistry $141322-328$.

Waberski D, Weitze KF, Lietmann C, Lübbert zur Lage W, Bortolozzo F, Willmen T \& Petzoldt R 1994a The initial fertilizing capacity of long-term-stored liquid boar semen following pre- and postovulatory insemination. Theriogenology 41 1367-1377.

Waberski D, Meding S, Dirksen G, Weitze KF, Leiding C \& Hahn R 1994b Fertility of long-term stored boar semen: influence of extender (Androhep and Kiev), storage time and plasma droplets in the semen. Animal Reproduction Science 36 145-151.

Wagner A, Ekhlasi-Hundrieser $M$, Hettel C, Petrunkina A, Waberski D, Nimtz M \& Töpfer-Petersen E 2002 Carbohydratebased interactions of oviductal sperm reservoir formation-studies in the pig. Molecular Reproduction and Development $\mathbf{6 1}$ 249-257.

Watson PF 1996 Cooling of spermatozoa and fertilizing capacity. Reproduction in Domestic Animals 31 135-140.

Received 21 May 2005

First decision 22 July 2005

Revised manuscript received 19 September 2005

Accepted 11 October 2005 